\title{
Diamond Burr for the Treatment of an Indolent Corneal Ulcer in a Foal
}

\author{
João Antonio Tadeu Pigatto, Luciane de Albuquerque, Ângela Beatriz de Oliveira Bacchin, \\ Géssica Maria Ribeiro da Silva, Michelle Becker Petersen \& Gabriela Grandi Reiter
}

\begin{abstract}
Background: Indolent corneal ulcers have been described as superficial ulcers with an associated rim of loose peripheral epithelium Treatment for indolent ulcers include debridement, grid keratotomy, multiple punctate keratotomy, third eyelid flaps, application of cyanoacrylate tissue adhesives, superficial keratectomy, and a debridement with a diamond burr.

Case: A 2-month-old female American Quarter Horse was referred to the Ophthalmology Veterinary Section of the Federal University of Rio Grande do Sul (UFRGS), Porto Alegre, Brazil, presenting epiphora and blepharospasm. A local veterinarian doctor had prescribed broad spectrum topical antibiotic and anti-inflammatory drops, although there was no positive response to the treatment for the past two weeks. Ophthalmic examination reveals and moderate discomfort in the left eye, epiphora, and mild corneal edema in the area of the defect. Slit lamp biomicroscopy revealed a superficial corneal ulcer with about $6 \mathrm{~mm}$. Corneal epithelium did not adhere to underlying corneal stroma. The remainder of the ophthalmic examination of the left eye was unremarkable. The diagnosis of a corneal ulcer was made based on these clinical signs and fluorescein staining of the cornea where the stain dissects under the unattached epithelial lip. A handheld battery-operated motorized diamond burr, with a $3.5 \mathrm{~mm}$ medium grit tip, was utilized to remove the epithelium. The medical treatment included tobramycin eye drops, and flurbiprofen sodium ophthalmic solution, being applied six times daily, after the procedure, during two weeks, and atropine sulphate $1 \%$ was applied once a day, during three days. The foal was hospitalized until healing the corneal ulcer. For two weeks, the foal was assessed daily, and, after that, follow-up visits were scheduled weekly for four months. Healing was defined as the point at which the cornea no longer retained fluorescein.

Discussion: Ulcers localized to the corneal epithelium, do not heal within the expected time frame, and have been characterized by epithelial border poorly adherent corneal and being commonly referred as indolent corneal ulcers. In this case, corneal ulcers are chronic and have not responded to an appropriate therapy for 14 days. The corneal ulcer was diagnosed based on history, clinical signs and fluorescein staining of the cornea. Usually, medical treatments provide disappointing results. Surgical treatment aims to remove the loosened epithelium in order to facilitate the growth of new epithelial cells, with stronger adhesion complexes. The utilization of a diamond burr, for the treatment of an indolent corneal ulcer, has been previously reported in humans, and dogs. In the present case, the debridement with a diamond burr was chosen on account of excellent results obtained in previous studies in humans and dogs, when treating indolent corneal ulcers. In the current case, the corneal ulcer healed in ten days, while in a study comparing the outcome in 23 horses treated by debridement, grid keratotomy, or superficial keratectomy, the mean times until complete healing were 15, 16 and 23 days, respectively. In the present case, debridement with diamond burr was effective in the treatment of a recurrent corneal ulcer in a foal.
\end{abstract}

Keywords: equine, recurrent corneal erosions, diamond burr. 


\section{INTRODUCTION}

Ulcers that are limited to the corneal epithelium and do not heal within the expected time frame, presenting poorly adherent corneal epithelium, which may be easily removed they are commonly referred as non-healing corneal ulcers [1,5]. The diagnosis of a non-healing superficial corneal ulcer is based on the present some redundant non-adhered epithelial border feature, being the lesion limited to epithelial loss, with little to no corneal stromal involvement $[2,4,9,10]$. The response to conservative topical therapy has been predictably poor, while the best chosen treatment has been the surgical procedure. In dogs, debridement, grid keratotomy, multiple punctate keratotomy, third eyelid flaps, application of cyanoacrylate tissue adhesives, superficial keratectomy, and a debridement with a diamond burr have been the utilized. The mentioned surgical procedures in horses, include thermal cautery, debridement, grid keratotomy and superficial keratectomy $[2,4,6,9]$. In 1983, a diamond burr was first used for the treatment of corneal erosions in humans [14]. Based on information available in the literature, this is the first case of an indolent corneal ulcer in a foal treated by using a diamond burr.

\section{CASE}

A 2-month-old female Quarter horse was referred to the Ophthalmology Section in the Veterinary Clinics Hospital of the Federal University of Rio Grande do Sul (UFRGS), Porto Alegre, Brazil, presenting epiphora and mild blepharospasm. The cause of the initial ulcer was unknown. A local veterinarian doctor had prescribed broad spectrum topical antibiotic and anti-inflammatory drops, although there was no positive response to the treatment for the past two weeks. Ophthalmic examination reveals and moderate discomfort in the left eye and mild corneal edema in the area of the defect. Slit lamp biomicroscopy (Portable Slit Lamp SL 15) ${ }^{1}$ revealed a superficial corneal ulcer with about $6 \mathrm{~mm}$. The diagnosis of a corneal ulcer was made based on these clinical signs and fluorescein (Fluorescein strips) $)^{2}$ staining of the cornea. Fluorescein stain under the non-adherent epithelium at the margins of ulcer (Figure 1). A battery-operated handheld diamond burr unit with a $3.5 \mathrm{~mm}$ round diamond drill (Algerbrush) ${ }^{3}$ was utilized to remove the epithelium. Moreover, the patient was gently manually. The foal was submitted to manual restraint and maintained in lateral recumbency. After the sterile preparation and draping, a wire speculum was utilized to increase the exposure of the cornea, and topical anesthetic solution (Anesthesic eye drops) ${ }^{4}$ was administered prior to the debridement. The diamond burr was passed over the ulcer, removing the non-adherent epithelial tissue, until some stable epithelium was found, and some uniform surface was obtained. The wounded area was debrided for $30 \mathrm{~s}$. The medical treatment included tobramycin eye drops (Tobrex) $)^{5}$ and flurbiprofen sodium ophthalmic solution (Ocufen) ${ }^{4}$ being applied 6 times daily, after the procedure, during 2 weeks, and atropine sulphate $1 \%$ (Atropine eye drops $1 \%)^{4}$ was applied once a day, during 3 days. On the first day, after surgery, the eye was visual, and the foal showed no clinical signs of ocular discomfort. In $72 \mathrm{~h}$, the condition of the eye remained stable, with no evidence of infection, inflammatory reaction or ocular discomfort. After 10 days, the fluorescein staining was negative (Figure 2). There was no recurrence of the ulcer after 4 months.

\section{DISCUSSION}

The features of non-healing superficial corneal ulcers are non-infected epithelial defects chronic and have not responded to an appropriate therapy for 2 weeks without stromal loss $[7,8]$. The lesion was previously reported in dogs, cats, elephant, llama, and horses $[1-3,8,13]$. In the current case the corneal ulcer was diagnosed based on history, clinical signs, fluorescein staining of the cornea and the ulcer was being unsuccessfully treated for 2 weeks. Indolent corneal ulcers have been described as superficial ulcers with an associated rim of loose peripheral epithelium and in spite of chronicity, vascularization typically minimal $[4,5,10]$.

The clinical findings of this case were similar to those previously reported. Usually, medical treatments provide disappointing results. The surgical treatment aims to remove the loosened epithelium in order to facilitate the growth of new epithelial cells, with stronger adhesion complexes [2-4]. There are few reports of indolent ulcers in horses documented in the literature $[2,4,6,9]$.

The utilization of a diamond burr, for the treatment of an indolent corneal ulcer, has been previously reported in humans and dogs $[7,12,14]$. In the present case, the debridement with a diamond burr was chosen on account of excellent results obtained in previous studies in humans and dogs $[7,14]$. Furthermore, this procedure is fast, safe and general anesthesia of the 


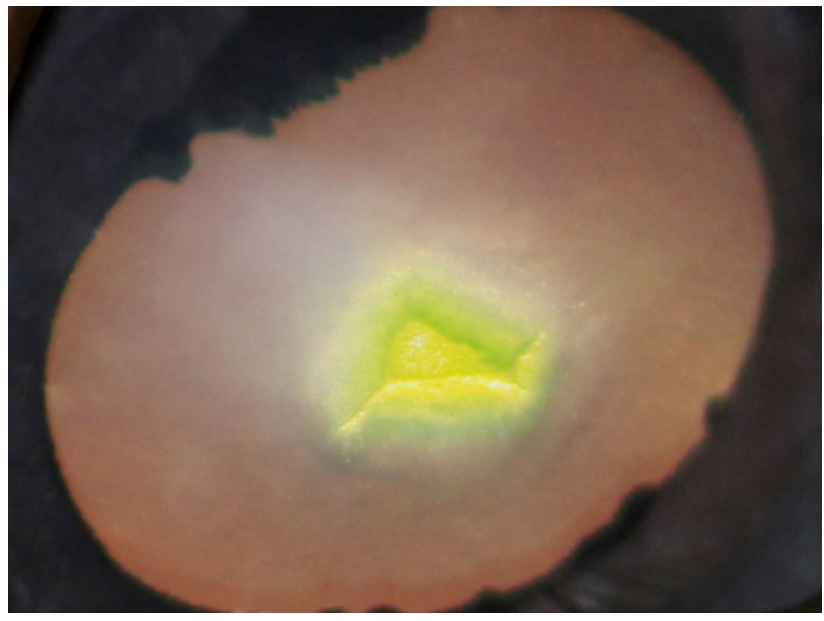

Figure 1. Left eye of a foal with a nonhealing corneal ulcer. Fluorescein migrated under the loose epithelium.

patient is not required. In the current case, the procedure was performed only under topical anesthesia. The procedure was done only with mechanical containment of the foal and drops topical anesthetic. This protocol has already been successfully applied in the treatment of corneal ulcer with cyanoacrylate in a foal [11].

The surgical treatment aims to remove the abnormal epithelial basement membrane and provide some smooth surface for facilitating the growth of new epithelial cells with stronger adhesion complexes $[3,10]$. In this case, the wounded area was debrided until some stable epithelium was found, and some uniform surface was then obtained. The corneal ulcer healed in 10 days, while in a study comparing the outcome in 3 horses treated by debridement, grid keratotomy, or superficial keratectomy, the mean times until complete healing were 15,16 and 23 days, respectively [9]. Although a mechanical corneal protection, with the third eyelid, or a bandage contact lens, was not utilized the foal showed no ocular discomfort in the

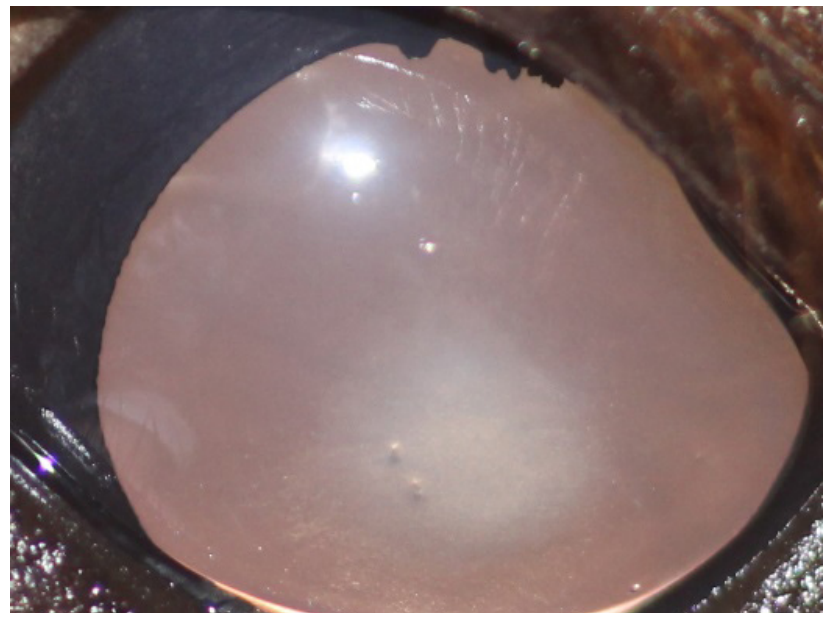

Figure 2. Left eye of a foal with a nonhealing corneal ulcer 10 days after diamond burr debridement. Corneal ulcer is healed.

postoperative period. Previous studies in humans and dogs have found low recurrence rate of the described disease, after utilizing a debridement with a diamond burr $[7,12,14]$. In this case, there was no recurrence of the ulcer during a during the 4-month period.

A debridement with a diamond burr is a simple procedure to perform, providing, overall, good, nonpainful, uncomplicated and rapid healing, without side effects and little scar tissue formation [7,12,14]. Debridement with the diamond burr was effective and safe for treating a recurrent corneal ulcer in a foal.

\section{MANUFACTURERS \\ ${ }^{1}$ Kowa Company Ltd. Isesaki Gunma-ken, Japan. ${ }^{2}$ Ophthalmos Indústria Farmacêutica. São Paulo, SP, Brazil. ${ }^{3}$ Alger Equipment Company Inc. Lago Vista, TX, USA. \\ ${ }^{4}$ Allergan Produtos Farmacêuticos LTDA. São Paulo, SP, Brazil. ${ }^{5}$ Alcon Laboratórios do Brasil. São Paulo, SP, Brazil.}

Declaration of interest. The authors report no conflicts of interest. The authors alone are responsible for the content and writing of the paper.

\section{REFERENCES}

1 Bentley E., Abrams G.A., Covitz D., Cook C.S., Fischer C.A., Hacker D., Stuhr C.M., Reid T.W. \& Murphy C.J. 2001. Morphology and immunohistochemistry of spontaneous chronic corneal epithelial defects (SCCED) in dogs. Investigative Ophthalmology \& Visual Science. 42(1): 2262-2269.

2 Bentley E. \& Murphy C.J. 2004. Thermal cautery of the cornea for treatment of spontaneous chronic corneal epithelial defects in dogs and horses. Journal of the American Veterinary Medical Association. 224(2): 250-253.

3 Bromberg N.M. 2002. Cyanoacrylate tissue adhesive for treatment of refractory corneal ulceration. Veterinary Ophthalmology. 5(1): 55-60.

4 Brunott A., Boeve M. \& Velden M. 2007. Grid keratotomy as a treatment for superficial nonhealing corneal ulcers in 10 horses. Veterinary Ophthalmology. 10(3): 162-167.

5 Burling K., Seguin M.A., Marsh P., Brinkman K., Madigan J., Thurmond M., Moon-Massat P., Mannis M. \& 
J.A.T. Pigatto, L. Albuquerque, A.B.O. Bacchin, G.M.R. Silva, M.B. Petersen \& G.G. Reiter. 2017. Diamond Burr for the

Murphy C.J. 2000. Effect of topical administration of epidermal growth factor on healing of corneal epithelial defects in horses. American Journal of Veterinary Research. 61(9): 1150-1155.

6 Cooley P.L. \& Wyman M. 1986. Indolent-like corneal ulcers in 3 horses. Journal of the American Veterinary Medical Association. 188(3): 295-297.

7 Gosling A.A., Labelle A.L. \& Breaux C.B. 2013. Management of spontaneous chronic corneal epithelial defects (SCCEDs) in dogs with diamond burr debridement and placement of a bandage contact lens. Veterinary Ophthalmology. 16(2): 83-88.

8 Jones M.L., Gilmour M.A. \& Streeter R.N. 2007. Use of grid keratotomy for the treatment of indolent corneal ulcer in a llama. The Canadian Veterinary Journal. 48(4): 416-419.

9 Michau T., Schwabenton B., Davidson M.G. \& Gilger B.C. 2003. Superficial, nonhealing corneal ulcers in horses: 23 cases (1989-2003). Veterinary Ophthalmology. 6(4): 291-297.

10 Morgan R.V. \& Abrams K.L. 1994. A comparison of six different therapies for persistent corneal erosions in dogs and cats. Veterinary and Comparative Ophthalmology. 4(7): 38-43.

11 Pigatto J.A.T., Hünning P.S., Rigon G.M., Soares M., Neumann C.F. \& Andrade M.C.C. 2012. Utilization of enbucrylate adhesive in the treatment of a corneal ulcer in a horse. Acta Scientiae Veterinariae. 40(4): 1092-1096.

12 Silva E.G., Powell C.C., Gionfriddo J.R., Ehrhart E.J. \& Hill A.E. 2011. Histologic evaluation of the immediate effects of diamond burr debridement in experimental superficial corneal wounds in dogs. Veterinary Ophthalmology. 14(5): 285-291.

13 Wolfer J. \& Rich P. 1992. Persistent corneal erosion in an Asian elephant. The Canadian Veterinary Journal. 33(5): 337-339.

14 Wong V.W.Y., Chi S.C.C. \& Lam F.R.C.S. 2009. Diamond burr polishing for recurrent corneal erosions: Results from a prospective randomized controlled trial. Cornea. 28(2): 152-156. 